Noname manuscript No.

(will be inserted by the editor)

\title{
C7 Multi-Messenger Astronomy of GW Sources
}

M. Branchesi - G. Woan • .

P. Astone - I. Bartos • A. Colla •

S. Covino - M. Drago - X. Fan •

S. Frasca - C. Hanna • B. Haskell •

J.S. Hazboun • I.S. Heng • D.E. Holz •

N.K. Johnson-McDaniel • I. D. Jones •

L. Keer • S. Klimenko - G. Kostas .

S.L. Larson - I. Mandel • M. Mapelli •

C. Messenger • G. Mazzolo •

A. Melatos - S. Mohanty - V. Necula •

M. Normandin - L. Obara - R. Opiela •

B. Owen - C. Palomba - G.A. Prodi .

V. Re · F. Salemi - T.L. Sidery •

M. Sokolowski • K. Schwenzer .

V. Tiwari - M.C. Tringali •

G. Vedovato - W. Vousden •

I. Yakushin • A. Zadrony • B.M. Ziosi

Received: date / Accepted: date

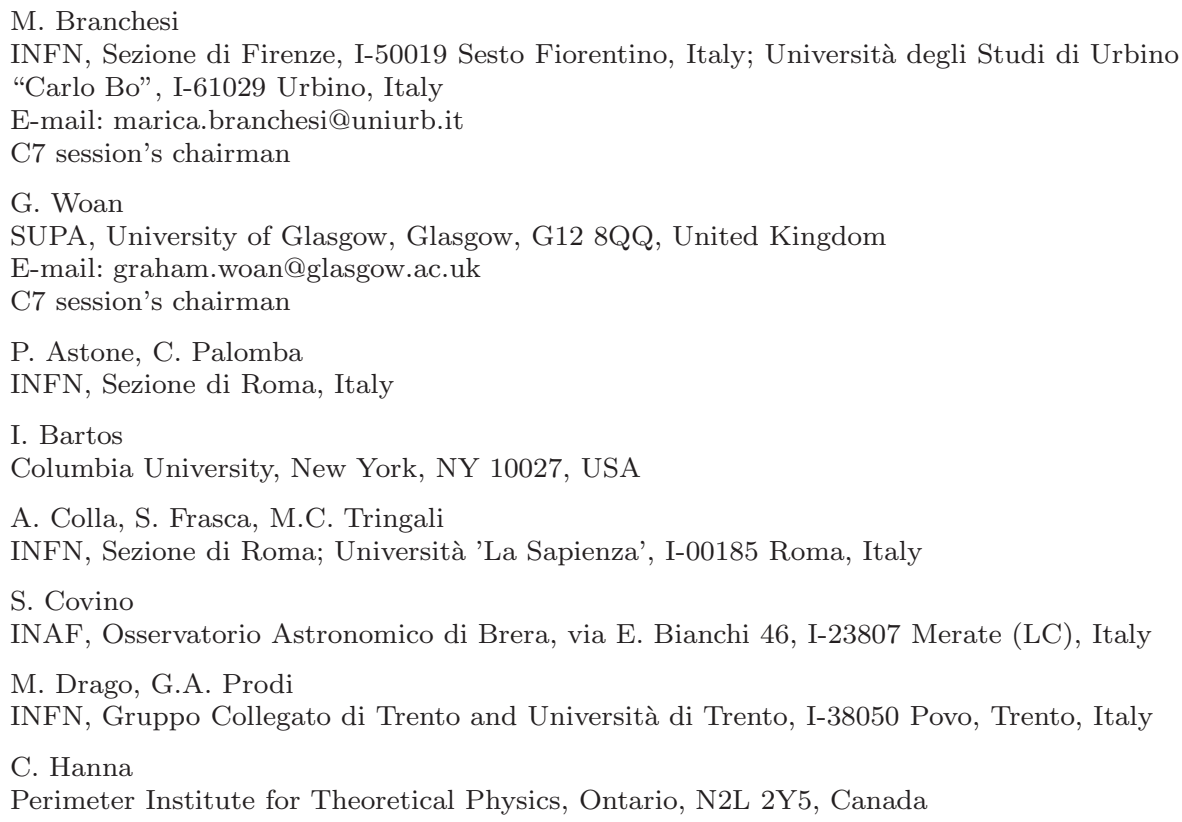


B. Haskell

University of Melbourne, Parkville, VIC 3010, Australia

J.S. Hazboun

Utah State University, Logan, Utah 84322

I.S. Heng, X. Fan, C. Messenger

SUPA, University of Glasgow, Glasgow G12 8QQ, United Kingdom

D.E. Holz

Kavli Institute for Cosmological Physics, University of Chicago, Chicago, IL 60637, USA

N.K. Johnson-McDaniel

Theoretisch-Physikalisches Institut, Friedrich-Schiller-Universitat, Max-Wien-Platz 1, 07743 Jena, Germany

L. Keer, I.D. Jones

University of Southampton, Southampton SO17 1BJ, United Kingdom

S. Klimenko, V. Necula, V. Tiwari

University of Florida, Gainesville, FL 32611, USA

G. Kostas

University of Murcia. Murcia, E-30100. Spain

S.L. Larson

CIERA \& Department of Physics and Astronomy, Northwestern University, Evanston, IL 60208

I. Mandel, T.L. Sidery, W. Vousden

University of Birmingham, Birmingham, B15 2TT, United Kingdom

M. Mapelli

INAF, Osservatorio Astronomico di Padova, Vicolo dell'Osservatorio 5, I-35122, Padova, Italy

G. Mazzolo, F. Salemi

Albert-Einstein-Institut, Max-Planck-Institut fur Gravitationsphysik, D-30167 Hannover, Germany and Leibniz Universitat Hannover, D-30167 Hannover, Germany

A. Melatos

University of Melbourne, Parkville, VIC 3010, Australia

S. Mohanty, M. Normandin

The University of Texas at Brownsville, Brownsville, TX 78520, USA

L. Obara

Faculty of Physics, University of Warsaw, Warsaw, Poland

R. Opiela

Centre for Theoretical Physics of the Polish Academy of Sciences, Warsaw, Poland

B. Owen

The Pennsylvania State University, University Park, PA 16802, USA

V. Re

INFN, Sezione di Roma Tor Vergata; Università di Roma Tor Vergata, I-00133 Roma

M. Sokolowski

National Center for Nuclear Research, Swierk, Poland; International Centre for Radio Astronomy Research - Curtin University, Perth, Australia; ARC Centre of Excellence for Allsky Astrophysics (CAASTRO)

K. Schwenzer

Washington University, St. Louis, Missouri, 63130, USA G. Vedovato

INFN, Sezione di Padova, I-35131 Padova, Italy 
Abstract We summarise the parallel session C7 Multi-Messenger Astronomy of $G W$ Sources in the GR20 Amaldi 10 Conference. The talks in this session covered a wide range of topics in multi-messenger astronomy.

Keywords multi-messenger $\cdot$ gravitational waves $\cdot$ electromagnetic emission

\section{Introduction}

The field of multi-messenger astronomy is entering a new and exciting era. The LIGO and Virgo gravitational-wave detector upgrades will soon be completed, with the expectation that they will have sufficient sensitivities to directly observe gravitational-wave (GW) signals for the first time. Just as multiwavelength electromagnetic (EM) observations have revolutionized our view of the Universe by probing the physical processes acting in astrophysical sources and unveiling new source classes, it is widely expected that the further inclusion of GW observations will open up a whole new set of possibilities.

From this perspective, the session C7 on Multi-Messenger Astronomy of $G W$ Sources highlights the importance of joint studies involving GW, EM and theoretical studies. The talks reflect the opportunities and challenges that multi-messenger observations offer, and the increasing importance of multimessenger techniques to interpreting astronomical data.

Several talks involved the most promising sources detectable by LIGO and Virgo: the coalescence of binary neutron stars and/or black holes. Binary merger rates are somewhat uncertain and consequently so is the expected rate of GW detections. If we assume that mergers of two NSs or a NS with a stellar BH are the progenitors of short gamma-ray bursts (GRBs), then the corresponding GW detection rate can be inferred from the short GRB observation rate (see 2). From another perspective, the same rate can be estimated by numerical simulations of compact object populations (see 2). Once GW signals are observed, the comparison between the short GRB rate, the GW detection rate and the estimated rates from simulations will constrain the theoretical models which govern the birth and the evolution of compact objects.

For a more complete understanding of the GW sources it would be extremely valuable to detect their electromagnetic counterparts. The main challenge here is the relatively poor sky localization offered by gravitational-wave detectors (tens of square degrees). This will require optimal observational strategies that involve telescope networks (see 2), galaxy targeting (see 2)

I. Yakushin

LIGO - Livingston Observatory, Livingston, LA 70754, USA

\section{A. Zadrozny}

National Center for Nuclear Research, Swierk, Poland

B.M. Ziosi

Department of Physics and Astronomy 'Galileo Galilei', University of Padova, Vicolo dell'Osservatorio 3, I-35122, Padova, Italy; INAF, Osservatorio Astronomico di Padova, Vicolo dell'Osservatorio 5, I-35122, Padova, Italy 
and the use of wide field telescopes (see 3), as well as the development of techniques to improve the sky localization (see $3,3,3,3$ ). There are special occasions (e.g., the G2 cloud falling towards the Galactic center, see 2) that offers a great opportunity to study and characterize GW source populations.

The importance of combining GW and and EM data is demonstrated by the possibility of evaluating the individual white-dwarf mass (see 2) using optical data from Gaia and the next-decade GW space detectors, such as LISA or NGO/eLISA. Other interesting examples come from the studies of low-mass X-ray binaries (see 2, 2,), oscillations in magnetar flares (see 3) and pulsar oscillations and glitches (see 2 and 3 ). In these cases the complementarity of information carried by electromagnetic and gravitational waves will shed new light on neutron star interiors and crustal physics.

The EM observations of pulsars are crucial to perform targeted searches to detect the continuous GW signal expected from non-pulsing neutron stars (see 2.7). Conversely, established GW search techniques turn out to be very effective at detecting new pulsars in gamma-ray data (see 3). Finally, GW/EM multi-messenger astronomy will be crucial to test the different theories of gravity (see 2).

\section{Contributed talks}

\subsection{GRB beaming and gravitational-wave observations (Presenter: D. Holz)}

Using the observed rate of short-duration gamma-ray bursts (GRBs) it is possible to make predictions for the detectable rate of compact binary coalescences in gravitational-wave detectors. We make predictions for the rate of events in future networks of gravitational-wave observatories, finding that the first detection of a NS-NS binary coalescence associated with the progenitors of short GRBs is likely to happen within the first 16 months of observation, even in the case of only two observatories (e.g., LIGO-Hanford and LIGO-Livingston) operating at intermediate sensitivities (e.g., advanced LIGO design sensitivity, but without signal recycling mirrors), and assuming a conservative distribution of beaming angles (e.g., all GRBs beamed within $30 \mathrm{deg}$ ). Less conservative assumptions reduce the waiting time to a period of weeks to months. Alternatively, the compact binary coalescence model of short GRBs can be ruled out if a binary is not seen within two years by a three detector network at advanced design sensitivity. We demonstrate that the gravitational-wave detection rate of GRB triggered sources (i.e., those seen first in gamma rays) is lower than the rate of untriggered events (i.e., those seen only in gravitational waves) if the beaming is less than $30 \mathrm{deg}$, independent of the noise curve, network configuration, and observed GRB rate. The first detection in gravitational waves of a binary GRB progenitor is therefore unlikely to be associated with the observation of a GRB. 


\subsection{Young dense star clusters: the nursery of double} compact-objects (Presenter: M. Mapelli)

Young star clusters (YSCs) are important for double compact object binaries (DCOs) for several reasons. First, YSCs are the nursery of stars: most stars $(\sim 80 \%,[1])$ form in YSCs. Thus, most compact object $(\mathrm{CO})$ progenitors form in YSCs. Furthermore, the most massive $\left(\gtrsim 10^{3} M_{\odot}\right)$ and densest $\left(\gtrsim 10^{3}\right.$ stars $\mathrm{pc}^{-3}$ ) YSCs are dynamically active environments: close encounters between single stars and binary systems in YSCs are extremely common and alter the orbital properties of the binaries ([2] and references therein). One of the most frequent outcomes of three-body encounters are dynamical exchanges, during which the single star replaces one of the former members of the binary and becomes member of the binary. The probability for an exchange to occur is particularly high if the mass of the single star is similar to (or higher than) the mass of one of the members of the binary. Since black holes (BHs) are among the most massive objects in star clusters (SCs) with an age $\gtrsim 10$ Myr, dynamical exchanges are particularly important for the formation of $\mathrm{BH}$ binaries: a $\mathrm{BH}$ that was born from a single star in the field remains single, while a $\mathrm{BH}$ that was born from a single star in a SC likely acquires a companion through dynamical exchanges. Thus, in order to study the demographics of DCOs, we need to account for the fact that most COs form and dynamically evolve in YSCs.

To this purpose, we ran $600 \mathrm{~N}$-body realizations of $\mathrm{YSCs}^{1}$, to study the formation and evolution of $\mathrm{BH}-\mathrm{BH}$ binaries, $\mathrm{BH}$-neutron star (NS) binaries and NS-NS binaries. Our simulations were performed with the STARLAB software environment [3], which couples a direct $N$-body integrator with recipes for stellar evolution (adopting the version presented in [2]). Our main results are the following (see [4], for details). The BH-BH binaries that form in our simulations are $\gtrsim 10$ times more numerous than NS-NS and BH-NS binaries. This is an effect of dynamics, since BHs are a factor of 3-4 less numerous than NSs, but the vast majority $(\sim 97 \%)$ of $\mathrm{BH}-\mathrm{BH}$ binaries form by exchange. On the other hand, $\mathrm{BH}-\mathrm{BH}$ binaries span a wide range of orbital periods (from $\sim 10^{-3}$ to $10^{7} \mathrm{yr}$ ) and most of them will never become important sources of gravitational waves (GWs), since their timescale for coalescence by GW emission is larger than the Hubble time. In particular, we expect $\sim 3(0) \mathrm{BH}-\mathrm{BH}$ mergers per 200 YSCs in 1.5 Gyr at metallicity $Z \leq 0.1 \mathrm{Z}_{\odot}\left(Z=\mathrm{Z}_{\odot}\right)$. Instead, NS-NS binaries are less numerous, but most of them have orbital periods $<1$ yr and are expected to merge in less than a Hubble time. We find $\sim 8(2)$ NS-NS mergers per 200 YSCs in $0.1 \mathrm{Gyr}$ at $Z=1 \mathrm{Z}_{\odot}\left(Z \leq 0.1 \mathrm{Z}_{\odot}\right)$. This indicates that NS-NS mergers are enhanced at solar metallicity, while $\mathrm{BH}-\mathrm{BH}$ mergers are more frequent at sub-solar metallicity. Finally, a large portion of COs ( $\sim 90 \%$ and $\sim 40 \%$ of NSs and $\mathrm{BHs}$, respectively) are ejected from

\footnotetext{
1 We assume YSC mass $\sim 3500 \mathrm{M}_{\odot}$, virial radius $1 \mathrm{pc}$, a King profile with dimensionless central potential $W_{0}=5$ and three different metallicities: $0.01,0.1$ and $1 \mathrm{Z}_{\odot}$. Metallicity affects the mass range of BHs: BHs with mass up to 80,40 and $23 \mathrm{M}_{\odot}$ can form from progenitor stars with metallicity $Z=0.01,0.1$ and $1 \mathrm{Z}_{\odot}$, respectively.
} 
the parent SC in < 100 Myr, by either supernova kick or dynamical recoil. Even the remaining COs end up in the galaxy field, after SC tidal disruption. Thus, we find that most of the predicted DCO mergers occur in the field, after dynamical ejection or evaporation of the SC. This is consistent with recent studies of short gamma-ray burst location (e.g., [5]).

\subsection{Follow-up activities for the transient universe (Presenter: S. Covino)}

One of the most important aspects of modern observational projects is the need for efficient and reliable follow-ups. This is typically true for surveys, where one needs to better characterize a subset of the identified sources, but it is also true whenever one opens new observational windows and gravitationalwave astronomy is no exception. The first meaningful signals detected by the next generation of detectors will certainly require follow-up electromagnetic observations to give further confidence to the detections themselves. In addition to this, the discovery space of joint GW / electromagnetic astronomy is huge, and we are really at the dawn of a new scientific era.

Follow-up observations of GW sources is unfortunately a far from easy task. The error boxes associated with GW detections can be very large, up to a few $\times 100 \mathrm{deg}^{2}$, and the higher probability regions for the location of GW counterparts have a complex shape and are formed by many smaller boxes located at large angular scales. In order to manage this very demanding observational task we need a well-balanced mix of observational facilities, from the highest energies to the radio band, and a geographical coverage that allows one to follow events that might be unobservable from any specific site.

Given the size of the error boxes, large field-of-view (FOV) instruments are required. For a regular optical telescopes with FoV of a few $\times 10 \operatorname{arcmin}^{2}$ the time required to cover a typical $100 \mathrm{deg}^{2}$ error box would be of the order of a week. With a survey telescope with $1 \mathrm{deg}^{2}$ FoV the same scientific goal can be fulfilled in a few hours.

The only solution to organizing and managing a reliable follow-up system is therefore to negotiate agreements between the different communities. Given the wide range of technical and scientific skills required we should also seriously consider the creation of new facilities, managed directly by the consortia leading the GW instruments, such as robotic optical telescopes.

\subsection{Use of galaxy catalogs for following up GW detections from binary neutron star mergers using advanced detectors (Presenter: W. Vousden)}

The 10-100 $\mathrm{deg}^{2}$ localization error provided by gravitational-wave transient searches presents a challenging problem for astronomers hoping to promptly follow up gravitational-wave observations of binary neutron star mergers with electromagnetic instruments. It has been suggested that restricting observations to known galaxies within the search range may significantly reduce the 
area of sky that must be imaged; however, current and proposed follow-up surveys can take wide-field images with FOV of $\sim 0.1-10 \mathrm{deg}^{2}$, and in this scenario we can expect many galaxies per field-of-view.

We assess the utility of a galaxy catalog in directing such an EM follow-up in terms of two parameters: the mean number of galaxies per field-of-view, and the fractional coverage of the search area available with limited telescope time. Galaxies are assigned to a grid of telescope fields-of-view on the sky and the brightest tiles selected for follow-up. We also consider the effects of incompleteness in the galaxy catalog under the assumption of a simple flux limit.

The greatest gains can be seen for early advanced detector configurations with fewer relevant galaxies per field-of-view. Here, the probability of a successful follow-up might increase as much as four-fold for a well-localized source; for example, from $5 \%$ to $\sim 20 \%$ for a $1 \mathrm{deg}^{2}$ telescope covering a $20 \mathrm{deg}^{2}$ uncertainty region out to $100 \mathrm{Mpc}$.

Ultimately, while EM follow-up will inevitably be challenging, we show that the inherent fluctuations in the galaxy number per pointing do modestly improve the prospects of follow-up, even for realistic incomplete catalogs.

2.5 G2 can Illuminate the Black Hole Population near the Galactic Center (Presenter: I. Bartos)

Most galaxies host very high-mass, so-called supermassive black holes in their very center (e.g., [6]), with masses million-billion times greater than the mass of the Sun. These supermassive black holes are expected to be surrounded by a large population of stellar-mass black holes [7-10]. These black holes, formed during the deaths of massive stars around the galaxy, migrate towards the central supermassive black hole via dynamical friction at the expense of low-mass stars, which are pushed outward. As a result, stellar-mass black holes form a steep density cusp in the center, with the heaviest black holes getting the closest [7].

Some much heavier, so-called intermediate-mass black holes $\left(\sim 10^{2}-\right.$ $10^{6} \mathrm{M}_{\odot}$ ) may also be formed through various processes. They can be created during the collapse of Population III stars in the early universe, as a result of runaway collisions of stars, or via the merger of smaller, stellar mass black holes ([11] and references therein). These larger black holes may also move towards the galactic center. If they have enough time to approach the center, they will experience the same dynamical friction as stellar mass black holes, and, due to their larger mass, move to the closest orbits from the central supermassive black hole.

Despite the robust theoretical prediction of a large number of black holes expected near galactic centers $(\sim 20000$ in the Milky Way [7-9]), direct observation is difficult (e.g., [9]). Nevertheless, there are indications of the overabundance of black holes or neutron stars within the central parsec of Sgr A* $[12]$.

A solar-system-size gas cloud called G2 is currently heading towards Sgr A* in the center of the Milky Way on a highly eccentric orbit [13-15], represent- 
ing a unique opportunity of observing black holes closely orbiting the Galactic Center. The cloud is expected to reach the vicinity of the central black hole, to a distance as low as $2 \times 10^{15} \mathrm{~cm}$, where a part of it will accrete onto the black hole.

Towards the pericenter passage of G2, the stellar-mass black hole population is expected to reach a sufficiently high number density such that G2 is likely to encounter multiple black holes over a period of a few months around its pericenter passage [16,17]. Our results [15] show that, once G2 gets close enough to $\mathrm{Sgr} \mathrm{A}^{*}$, it is likely to encounter black holes, probably experiencing multiple encounters during its travel.

Upon an encounter, the black hole accretes gas from G2, converting some of the gravitational potential of the gas into multi-wavelength radiation. The strength of the emission will depend on the black-hole mass, the relative velocity of the black hole and G2, the cloud temperature and density, and the total angular momentum of the infalling gas. Using radiative efficiencies from the literature, we estimated the expected luminosity of an encounter. Comparing an encounter's expected X-ray luminosity to the sensitivity of the Chandra satellite to a source in the direction of $\mathrm{Sgr} \mathrm{A}^{*}$ for an observation period of 1 week, we calculated the detectability of an encounter as a function of black hole mass and relative velocity. Our results indicate that the detection of G2's encounter with stellar-mass black holes requires low relative velocities, therefore only a fraction of such encounters may be detectable. For intermediate mass black holes, encounters with black-hole mass $\gtrsim 5000 \mathrm{M}_{\odot}$, the majority of encounters will be detectable [15].

\subsection{Measuring the masses of white dwarf binaries - a multi-messenger approach (Presenter: N. Johnson-McDaniel)}

One can combine gravitational-wave and electromagnetic observations of white dwarf binaries to obtain the individual masses of the binaries components. This is a measurement that cannot be made using either messenger alone, in practice, except in certain exceptional situations (or by invoking considerable modelling). The authors evaluate the anticipated accuracy of this measurement for the binaries in a population synthesis model of the galaxy. In one possible scenario (using the parallax from Gaia and the mass function from spectroscopy), they find that one can measure the masses of hundreds of systems with accuracies of tens of percent, using classic LISA. This measurement would also be able to identify around 20 binaries as having a total mass above the Chandrasekhar mass (and thus being potential Type Ia supernova progenitors) at $2 \sigma$ confidence. The authors also consider NGO/eLISA with $1 \mathrm{Gm}$ and $2 \mathrm{Gm}$ arms and evaluate the properties of the subpopulation with accurate mass measurements for each of these detectors. The number of systems with accurate mass measurements decreases as the detector's sensitivity decreases, but even for NGO/eLISA with $1 \mathrm{Gm}$ arms, one can still measure the masses of $\sim 100$ systems with accuracies of a few percent. 
The authors also considered performing such measurements with only one electromagnetic observation. Such measurements rely on being able to measure the higher harmonics in the gravitational-wave signal, and are thus only applicable to a few strong sources, if at all. However, the authors found that one can measure at least one of the individual masses of about 5 high-frequency systems with an accuracy of better than $40 \%$ using classic LISA and the Gaia parallax measurement. For the analogous case with classic LISA and only a mass function measurement, the individual mass measurements would be quite inaccurate, in practice. For all of these measurements, the authors showed that finite size effects will not contaminate this measurement at the accuracies obtainable for the systems in the population model they used.

\subsection{Astronomical guidance for directed searches for continuous gravitational waves (Presenter: B. Owen)}

The LIGO Scientific Collaboration and Virgo Collaboration have published a search for continuous gravitational-waves from the non-pulsing neutron star in supernova remnant Cas A. More such searches, where the direction is known but no pulsar timing is available, are under way. I describe the astronomical criteria for good targets for such gravitational-wave searches, list classes of astronomical objects, and give examples of each class.

The main criterion is whether or not LIGO and Virgo have any hope of detecting the target consistent with energy conservation. An upper limit on gravitational-wave strain can be inferred from the age of and distance to the target, assuming that it has lost and continues to lose most of its energy and angular momentum in the form of gravitational waves [24]. If this number is high enough to be beaten by a computationally feasible search, a detection may be possible. A secondary criterion is localization of the source on the sky so that a directed search may beat the sensitivity of all-sky searches. For the longest integration times arc second location is needed, but some searches can tolerate uncertainties of a few arc minutes.

Not surprisingly, the criteria favor young and nearby neutron stars or likely locations. Some x-ray point sources and small pulsar wind nebulae fit the criteria, and even a few small supernova remnants with no such marker inside. Other locations likely to include young neutron stars include massive young clusters of main sequence stars, especially those with magnetars as the latter are relatively young and neutron star formation tends not to occur solo. And since recent accretion can rejuvenate old neutron stars for purposes of gravitational-wave emission, globular clusters with high interaction rates may also be good places to look.

Examples of good targets are easily found: Other than Cas A, the central compact object in the Vela Junior supernova remnant is one x-ray point source well worth a search. A supernova remnant with no such object yet small enough to target is SNR G1.9+0.3, recently taking the title of youngest known remnant in the galaxy from Cas A. Westerlund 1 is the prototype young massive cluster, complete with young magnetar, and nearby globular cluster possibilities include NGC 6544. 
Even with old LIGO and Virgo data, more targets of all these types are interesting for directed searches, and in the era of advanced interferometers the list will grow.

\subsection{An Update on Magnetic Mountains as Advanced LIGO Sources (Presenter: A. Melatos)}

Low-mass X-ray binaries (LMXBs) are regarded as a promising target for Advanced LIGO, because the sharp cut-off in their spin distribution at $\sim 0.7 \mathrm{kHz}$ (measured from persistent pulsations and transient X-ray burst oscillations) can be explained satisfactorily by gravitational-wave stalling, if the neutron star's mass ellipticity satisfies $\epsilon \sim 10^{-8}$, a plausible number. Other scenarios, involving radiation pressure or self-adjusting disk-magnetosphere coupling near the corotation radius, can also explain the cut-off but are not discussed further here. There are several possible origins for an ellipticity of the above size, including dynamic processes (e.g., r-modes) and quasi-static processes like mountains formed by thermal gradients (which drive nuclear reactions like electron capture at nonuniform rates), magnetic stresses inside the star and on the surface, and exotic components in the nuclear equation of state (e.g., hyperons); see [18] for a summary. Thermal mountains are limited in size by the breaking strain of the elastic crust, but this restriction does not apply to magnetic mountains.

In an LMXB, a magnetic mountain forms by polar magnetic burial: accreting gas is funnelled magnetically onto the magnetic poles of the star. The resulting $\epsilon$ is $\sim 10^{4}$ times greater than naive force-balance estimates predict, because the magnetic field is compressed strongly at the equator (with $B \gg 10^{12} \mathrm{G}$ ), as the mountain slides sideways under its own weight, even though the magnetic dipole moment (which is dominated by $B$ at the pole) is reduced to $\sim 10^{26} \mathrm{G} \mathrm{cm}^{3}[18]$. In recent years, detailed analytic and numerical modeling of magnetic mountains has uncovered three important pieces of new physics. First, the mountains are hydromagnetically stable in two and three dimensions to interchange Parker and resistive tearing modes. This is a counterintuitive result which has nevertheless been verified with two independent numerical codes $[19,20]$. Second, the ellipticity depends strongly on the nuclear equation of state, covering the range $10^{-8} \leq \epsilon \leq 10^{-4}$ for adiabatic indices in the range $1 \leq \Gamma \leq 5 / 3[21]$. Third, the mountain relaxes resistively on the long ohmic time-scale, not the short time-scale associated with tearing modes and reconnection (that is, the geometric mean of the ohmic and Alfvén times). Resistive relaxation and subsidence into the dense interior reduce $\epsilon$ by a factor of $\sim 4$ at most for typical LMXB lifetimes and magnetic field strengths $[22,23]$.

A variety of multi-messenger experiments will be possible, once LMXB mountains are detected as gravitational-wave sources. The measured ellipticity, combined with X-ray measurements of the temperature, constrains the historical rate of relaxation and hence the electrical resistivity of nuclear matter at crustal densities. The electrical resistivity is determined by the impurity abundance, a quantity of interest in nuclear physics. Impurities also control the 
breaking strain of the elastic crust. Accretion-driven oscillations of a magnetic mountain show up simultaneously in the gravitational-wave spectrum and Xray light curve/polarization, raising the prospect of mapping the magnetospheric structure. Magnetic "hedges" (i.e., barriers of compressed magnetic field) left behind by burial can thermally insulate parts of the stellar surface during thermonuclear X-ray bursts, thereby affecting burst recurrence times and harmonic content. Finally, it can be shown (Priymak et al., in preparation) that cyclotron resonance scattering features can be exploited to discriminate between mountains of thermal and magnetic origin, for example by comparing the depths of the generic twin troughs of a cyclotron line with the ellipticity inferred from a gravitational-wave detection. Cyclotron lines also constrain the magnetic and spin orientation independently, reducing the computational expense of gravitational-wave searches.

\subsection{The r-mode instability and the interior physics of neutron stars: constraints from X-ray, UV and GW signals (Presenter: B. Haskell)}

Rapidly rotating neutron stars in low-mass X-ray binaries may be an interesting source of gravitational waves $(\mathrm{GWs})$. In particular, several modes of stellar oscillation may be driven unstable by GW emission, and this can lead to a signal that may be detectable by next generation GW detectors. Even without a direct GW detection, however, current X-ray and ultraviolet observations can constrain the physics of the r-mode instability. In particular the core temperatures inferred from the data would place many systems well inside the unstable region predicted by standard physical models. However, this is at odds with theoretical expectations. A system in this region would heat up due to the shear from the unstable mode and spin down rapidly as it emits GWs. If the saturation amplitude for the mode is large the duty cycle is very short, and one would not expect to 'catch' systems in the unstable region. If, on the other hand, the saturation amplitude is small, as theoretical calculations suggest [25], then systems would never enter well into the unstable region. This is a clear indication that additional physics must be included in our models. One option is to investigate additional sources of damping which may act at lower temperatures [26,27]. These could, for example, be due to hyperons or deconfined quarks in the core. Other options include resonances with crustal modes, which lead to stronger damping at the crust/core interface for certain frequencies, or resonances with other inertial modes, which lead to stronger damping at specific temperatures [28]. Finally strong mutual friction may lead to the additional damping required to explain the observations. This is an interesting possibility, as strong mutual friction is to be expected if the protons in the outer core of the star are in a type II superconducting state. In this case the interaction between superfluid vortices and superconducting flux tubes will lead to strong damping. In fact the damping is strong enough that it may provide a saturation mechanism for the r-modes, as vortices will initially be pinned and not have enough energy to cut through the flux tubes. As the mode amplitude grows the relative flow between the superfluid and the 
charged component will lead to a Magnus force strong enough to unpin the vortices. At this point the strong damping will effectively halt the growth of the r-mode. Estimates of the saturation amplitude given by this mechanism suggest it may act before the mode saturates due to non-linear couplings [29]. This is an interesting possibility and allows for an alternative explanation of the observations, i.e., that the saturation amplitude could be low enough that the r-mode instability does not affect the spin and thermal evolution of the star, thus allowing a system to 'live' in the unstable region.

2.10 Neutron star oscillations from starquakes (Presenter: L. Keer)

Glitches - sudden increases in spin rate - are observed in many pulsars. One mechanism that has been proposed to account for these is the starquake model, in which glitches are triggered by a loss of strain in the solid crust of the star. Starquakes can be expected to excite some of the oscillation modes of the neutron star. These oscillations are of interest both as a source of gravitational waves and as a mechanism for modulating the radio emission.

The talk started with a discussion of some order-of-magnitude estimates for the amplitude of these oscillations and the corresponding gravitational-wave emission, and then moved on to describing a more detailed toy model that the authors are developing. In this, the star is modelled as solid and incompressible, allowing much of the calculation to be carried out analytically. The mechanism specified for the starquake is an instantaneous removal of strain from the whole star, while keeping the positions of particles in the star unchanged. Although this acausal mechanism is perhaps not very realistic, it is a useful place to start developing the model. Given this mechanism, initial data for the starquake can be calculated, and projected against a basis of normal modes of the star after the glitch, in order to find the amplitude of the modes excited. The results of this are reviewed for the special case where the star spins down to zero angular velocity before the starquake; most of the energy released in the quake is found to go into exciting a mode of the star similar to the f-mode of a purely fluid star.

2.11 Limiting bimetric theories of gravity and massive gravity theories with multi-messenger astronomy of pulsars (Presenter: J. Hazboun)

The advent of gravitational-wave astronomy provides new proving grounds for testing theories of gravity. Recent work has reinvigorated the study of bimetric theories of gravity and massive graviton theories [30,31]. Certain versions [32, 33], with an effective graviton mass as small as $10^{-49} \frac{\mathrm{eV}}{c^{2}}$, can account for the acceleration of the expansion of the universe without the need for dark energy. One of the most interesting predictions of these theories is the sub-luminal speed of propagating gravitational waves. Multi-messenger astronomy provides a unique opportunity to put limits on the difference between the propagation speed of electromagnetic and gravitational waves from these sources. Here we consider two multi-messenger cases: first, the limits from isolated pulsars based on the current best limits from LIGO [34] on gravitational-wave emission, and 
second the limits from ultra-compact binaries that will be visible to a lowfrequency space-based gravitational-wave observatory like LISA. The required phase comparison between the electromagnetic signal and the gravitationalwave signal is derived, and assuming a null result in that comparison, the current bounds on emission are used to place limits on alternative theories that exhibit propagation delays. It is shown that these limits do not depend on the distance to the source, but are generically dependent on the observation time. For pulsars the eccentricity and principal moment of inertia also enter into the relationship limiting the difference of the propagation speed from $c$ $\left(\delta=\frac{\left|v_{g w}-v_{e m}\right|}{c}\right)$.

$$
\delta_{\text {pulsar }}=\beta 1.105 \times 10^{22}\left(S_{h} \times 1 \mathrm{~Hz}\right)^{\frac{1}{2}}\left(\frac{f_{\mathrm{gw}}}{1 \mathrm{~Hz}}\right)^{3}\left(\frac{I_{3}}{10^{38} \mathrm{~kg} \mathrm{~m}^{2}}\right)^{-1}\left(\frac{e}{10^{-5}}\right)^{-1}\left(\frac{T}{1 \mathrm{yr}}\right)^{-\frac{1}{2}}
$$

Here $S_{h}$ is the spectral density of aLIGO and $\beta$ encodes uncertainty about the phase between the gravitational-wave source and the electromagnetic source. An analogous limit for binaries includes the chirp mass, where $S_{h}$ below is the spectral density of the LISA mission.

$$
\delta_{\text {binary }}=\beta 8.77 \times 10^{-13} M_{\odot}^{\frac{5}{3}} \frac{\left(M_{1}+M_{2}\right)^{\frac{1}{3}}}{M_{1} M_{2}}\left(\frac{10^{-3} \mathrm{~Hz}}{f_{0}}\right)^{\frac{11}{6}}\left(\frac{T}{1 \mathrm{yr}}\right)^{-\frac{1}{2}}\left(S_{h}^{\mathrm{SA}} \times 1 \mathrm{~Hz}\right)^{\frac{1}{2}}
$$

Observations of the pulsars in the most sensitive range of LIGO could put an upper limit on the graviton mass as low as $10^{-38} \frac{\mathrm{eV}}{c^{2}}$ and an upper limit on the percent difference between the waves speeds as low as $10^{-9}$; for a LISA-like mission the bounds are even better, $10^{-40} \frac{\mathrm{eV}}{c^{2}}$ and $10^{-12}$ respectively.

\section{Posters}

\subsection{Prompt searches for optical signal from gravitational wave transients with Pi of the Sky (Presenter: A. Zadrozny)}

Here we describe methods used by the Pi of the Sky team to search for optical counterparts of GW event candidates as a part of future Looc-Up observations, and to show the usefulness to such searches of telescopes with the large fieldsof-view. The Looc-Up project (2009-2010)[35,36] was initiated by the LSC and Virgo collaborations and several other electromagnetic (EM) observation teams. The project was aimed at finding such counterparts and performing an electromagnetic follow-up of the most promising GW event candidates selected by the low-latency analysis of the LIGO and Virgo detector data.

$\mathrm{Pi}$ of the Sky is a network of robotic telescopes dedicated to observations of short-timescale astrophysical phenomena, in particular optical counterparts of Gama Ray Bursts (GRBs). The network consists of two observatories located in different hemispheres (one in Chile and one in Spain) having fields-of-view of $400 \mathrm{deg}^{2}$ and $6000 \mathrm{deg}^{2}$, respectively. All Pi of the Sky telescopes are working 
with 10s exposure time. The telescope located in Spain can cover the Swift satellites most sensitive field-of-view in a single exposure.

During future joint GW-EM observations, the Spanish observatory will be able to image more than $30 \%$ of a GW candidate's error box in a matter of minutes, taking more than one picture of each observed field. What is more, the system can detect optical transients on-line, perform the automatic follow-up of interesting events and alert other, more sensitive telescopes.

\subsection{Factors limiting sky localization of gravitational-wave sources (Presenter: M. Drago)}

Electromagnetic counterparts are expected for some classes of gravitationalwave signals. Observation of such counterparts strongly depend on the sky localization capabilities of the GW detector networks. We study the source localization performances considering a coherent algorithm to search for unmodeled transient signals and we compare these results with the theoretical expectations from the triangulation method (i.e., based on differential measurements of the arrival time of the GW signal at the detectors). The pipeline achieves good sky localization for a large variety of injected waveforms at a mean SNR close to 10 for each detector, performing better than the simpler triangulation method. The performance is improved by adding more sites to the LIGO-Virgo detectors: typically a 4-fold network achieves one order of magnitude smaller sky error regions with respect to the standard 3-fold one.

\subsection{Reconstructing the sky position of coalescing binaries from gravitational-wave data using Bayesian methods (Presenter: T.L. Sidery)}

When the next generation of gravitational-wave interferometers come online we expect to follow up detections of coalescing compact binaries with electromagnetic observations. Accurate sky locations will be needed to help point these follow up searches. Sky location information can be quickly inferred from the relative time-delay of the arrival time of gravitational waves reaching each interferometer. While this is a fast approach, it does not coherently search over all the waveform parameters and so is using a limited set of the available information. A number of codes based on Bayesian analysis have been developed that search for the parameters of a source, including its sky position. The present work tries to identify the relative power and limitations of the two approaches in order to achieve the best science. While algorithms based just on triangulation are extremely fast compared to Bayesian codes, the comparison results suggest that the stochastic codes will give us a significant improvement in sky localisation and efforts to increase their efficiency are vital if they are able to compete with triangulation methods for being used in rapid electromagnetic followup. 
3.4 The impact of astrophysical information on sky localisation of gravitational waves in association with gamma-ray bursts

(Presenter: X. Fan)

We put forward a Bayesian inference framework which allowed information from electromagnetic observations to be incorporated into gravitational-wave data analyses for signal sky location estimation. In particular, we proposed the use of galaxy catalogues to reduce the uncertainty in the signal sky location, that is expected to be tens to hundred square degrees for the advanced LIGO and Virgo detector network. Our method shows that the use of the galaxy distribution as priors in the gravitational wave data analysis permits to improve the sky localization. Furthermore, preliminary tests of our proposed method show that the sky location uncertainity is further improved if properties of the host galaxy are included.

3.5 Towards optimal integration of multi-messenger information in a multi-trigger population study analysis (Presenter: M. Normandin)

We present an algorithm for population parameter estimation that optimally incorporates multi-messenger information for a set of triggers, such as gamma-ray bursts. Combining data from multiple triggers can increase the signal-to-noise and thus our belief in the association of GWs with the population. Significant improvement in the sensitivity of population study methods are expected from applying suitable weights that take into account the background population so that the triggers that are likely to contribute mostly to noise are suppressed in the analysis. In this context our method makes the background distribution to play a direct role in the parameter estimation procedure: we use a likelihood-based method where the multi-messenger data and the background astrophysical distribution are both incorporated into a common likelihood before the population parameters are estimated. To demonstrate the method, we present preliminary results of this method as applied to simulated triggers that contain combined electromagnetic and gravitational radiation.

\subsection{Magnetar burst oscillations and multi-messenger astronomy (Presenter: D.I. Jones)}

Quasi-periodic oscillations have been seen in the light curves following several magnetar giant flares. These oscillations are of great interest as they probably provide our first ever view of the normal modes of oscillation of neutron stars. The state-of-the-art lies in the study of the oscillations of elasticmagnetic stellar models, mainly with a view to relating the observed frequencies to the structure and composition of the star itself. We described how we advanced this programme by considering several new physical mechanisms that are likely to be important for magnetar oscillations. These relate to the superfluid/superconducting nature of the stellar interior, and the damping of the modes, both through internal dissipation mechanisms and the launching of 
waves into the magnetosphere. We made simple order-of-magnitude estimates to show that both the frequencies and the damping time of magnetar oscillations can evolve in time, identifying three distinct 'pathways' that can be followed, depending upon the initial magnitude of the mode excitation. These results are interesting as they show that the information buried in magnetar QPOs may be even richer than previously thought, and motivate more careful examination of magnetar light curves, to search for signatures of the different types of evolution that we have identified.

3.7 Probing compact star interiors with gravitational waves, radio waves and x-rays (Presenter: K. Schwenzer)

The rotation frequencies of young pulsars are systematically below their theoretical Kepler limit. Unstable r-mode oscillations, which emit copious gravitational waves and can thereby quickly spin down these sources, have been suggested as a possible explanation for this observation. We give an analytic solution for the pulsar evolution and the gravitational-wave emission due to r-modes [37], that allows us to assess this scenario and the uncertainties due to the underlying microphysics. We find that the frequency to which r-modes spin down a star is surprisingly insensitive to the microscopic details and the r-mode saturation amplitude. Comparing our results to astrophysical data we find that $\mathrm{r}$-modes provide a quantitative explanation for the observed small pulsars frequencies and that all observed young pulsars are very likely already outside the r-mode instability region. This requires a fast spindown implying a sufficiently large r-mode saturation amplitude. Therefore, unobserved young neutron stars associated with recent supernovae or pulsar wind nebulae present very promising sources for gravitational-wave searches. We show that advanced LIGO would be able to see several of them, like the neutron star in Cassiopeia A or a compact remnant of SN 1987A. Finally we find that despite the non-linear coupling of the thermal and spin-down evolution, the gravitational-wave signal is at sufficiently late times independent of the unknown r-mode saturation amplitude and depends on the unknown saturation mechanism only within some tens of per cent.

\subsection{Search of gamma-ray pulsars through the adaptation of methods used for the search of continuous GW signals (Presenter: M.C. Tringali)}

We have developed the initial semi-coherent step of a hierarchical method for the blind search of gamma-ray pulsars, based on techniques previously developed for the search of continuous gravitational-wave signals $(\mathrm{CW})$ from spinning neutron stars.

In both cases, the received signal is not exactly periodic due to the Doppler modulation, spin-down and relativistic effects, that must be accurately corrected. To this purpose we have adapted a computationally efficient timedomain procedure routinely used in the search of CW signals. A second fundamental ingredient is the construction of the search grid in the space of 
position and spin-down. The analysis is carried on each point of the grid and potentially interesting candidates are selected on the base of the value of a detection statistic computed from the pulse spectrum.

We have successfully tested the procedure using a sample of five gammaray pulsars recently discovered by the Fermi/LAT detector. The development of the candidate follow-up stage will be the subject of future work.

Acknowledgements M.Branchesi, B.M. Ziosi and M. Mapelli acknowledge financial support from the Italian Ministry of Education, University and Research (MIUR) through grant FIRB 2012 RBFR12PM1F. B.M. Ziosi acknowledges financial support from Padova University through Strategic Project AACSE. M. Mapelli acknowledges financial support from INAF through grant PRIN-2011-1. B.M. Ziosi and M. Mapelli acknowledge the CINECA Award N. HP10B3BJEW, HP10CLI3BX, HP10CXB7O8, HP10C894X7, HP10CGUBV0, HP10CP6XSO and HP10C3ANJY for the availability of high performance computing resources and support. The work of Adam Zadrozny was supported by Polish Ministry of Science and Higher Education grant no. DPN/N176/VIRGO/2009. This work has been partially financed by the Polish Ministry of Science and Higher Education in 2009-2013 as a research project and by POLISH-SWISS ASTRO PROJECT cofound under the Swiss program of cooperation with new member states of European Union.

\section{References}

1. Lada C.J. \& Lada E.A.: Embedded Clusters in Molecular Clouds. ARA\&A 41, 57-115 (2003)

2. Mapelli M., Zampieri L., Ripamonti E. \& Bressan A.: Dynamics of stellar black holes in young star clusters with different metallicities - I. Implications for X-ray binaries. MNRAS 429, 2298-2314 (2013)

3. Portegies Zwart S.F., McMillan S.L.W., Hut P. \& Makino J.: Star cluster ecology - IV. Dissection of an open star cluster: photometry. MNRAS, 321, 199-226 (2001)

4. Ziosi B.M., Mapelli M., Branchesi M. \& Tormen G.: Dynamics of stellar black holes in young star clusters with different metallicities - II. Black hole-black hole binaries. MNRAS 441, 3703-3717 (2014)

5. Fong W. \& Berger E.: The Locations of Short Gamma-Ray Bursts as Evidence for Compact Object Binary Progenitors. ApJ 776, 18-33 (2013)

6. Antonucci R.: Unified models for active galactic nuclei and quasars. ARA\& 31, 473-521 (1993)

7. Morris M.: Massive star formation near the Galactic center and the fate of the stellar remnants. ApJ 408, 496-506 (1993)

8. Miralda-Escudé J. \& Gould A.: Cluster of Black Holes at the Galactic Center. ApJ 545, 847-853 (2000)

9. Freitag M., Amaro-Seoane P. \& Kalogera V.: Stellar Remnants in Galactic Nuclei: Mass Segregation. ApJ 649, 91-117 (2006)

10. O'Leary R. M., Kocsis B. \& Loeb A.: Gravitational waves from scattering of stellar-mass black holes in galactic nuclei. MNRAS 395, 2127-2146 (2009)

11. Kocsis B., Ray A. \& Portegies Zwart S.:Mapping the Galactic Center with Gravitational Wave Measurements Using Pulsar Timing. ApJ 752, 67-77 (2012)

12. Muno M. P., Pfahl E., Baganoff F. K., Ghez, A. Lu, J. \& Morris M. R.: An Overabundance of Transient X-Ray Binaries within 1 Parsec of the Galactic Center. ApJ 622, L113-L116 (2005)

13. Gillessen S., Genzel R., Fritz T.K., Quataert E., Alig C., Burkert A., Cuadra J., Eisenhauer F., Pfuhl O., Dodds-Eden K., Gammie C.F. \& Ott T.: A gas cloud on its way towards the supermassive black hole at the Galactic Centre. Nature 481, 51-54 (2012) 
14. Burkert A., Schartmann M., Alig C., Gillessen S., Genzel R., Fritz T.K. \& Eisenhauer F.: Physics of the Galactic Center Cloud G2, on Its Way toward the Supermassive Black Hole. ApJ 750, 58-74 (2012)

15. Gillessen S., Genzel R., Fritz T.K., Eisenhauer F., Pfuhl O., Ott T., Cuadra J., Schartmann M. \& Burkert A.: New Observations of the Gas Cloud G2 in the Galactic Center. ApJ 763, 78-87 (2013)

16. de la Fuente Marcos R. \& de la Fuente Marcos C.: Colliding with G2 near the Galactic Centre: a geometrical approach. MNRAS 435, L19-L23 (2013)

17. Bartos I., Haiman Z., Kocsis B., Márka S.: Molecular clouds H2 clouds dense clouds and dark clouds, X-ray, Black holes, Galactic center bar circumnuclear matter and bulge. PhysRevLett 110, 221102 (2013)

18. Melatos A. \& Payne D.J.B.: Gravitational Radiation from an Accreting Millisecond Pulsar with a Magnetically Confined Mountain. ApJ 623, 1044-1050 (2005)

19. Vigelius, M. \& Melatos, A.: Three-dimensional stability of magnetically confined mountains on accreting neutron stars. MNRAS 386, 1294-1308 (2008)

20. Mukherjee D., Bhattacharya D. \& Mignone A.: MHD instabilities in accretion mounds - II. 3D simulations. MNRAS 435, 718-727 (2013)

21. Priymak M., Melatos A. \& Payne D.J.B.: Quadrupole moment of a magnetically confined mountain on an accreting neutron star: effect of the equation of state. MNRAS 417, 2696-2713 (2011)

22. Vigelius M. \& Melatos A.: Resistive relaxation of a magnetically confined mountain on an accreting neutron star. MNRAS 395, 1985-1998 (2009)

23. Wette K., Vigelius M. \& Melatos A.: Sinking of a magnetically confined mountain on an accreting neutron star. MNRAS 402, 1099-1110 (2010)

24. Wette K., Owen B.J., Allen B., Ashley M., et al.: Searching for gravitational waves from Cassiopeia A with LIGO. CQG 25, 235011 (2008)

25. Bondarescu R., Teukolsky S.A., Wasserman I.: Spin evolution of accreting neutron stars: Nonlinear development of the r-mode instability. Phys.Rev.D 76, 06401 (2007)

26. Ho W.C.G., Andersson N., Haskell B.: Revealing the Physics of r Modes in Low-Mass X-Ray Binaries. Phys.Rev.Lett. 107, 101101 (2011)

27. Haskell B., Degenaar N., Ho W.C.G.: Constraining the physics of the r-mode instability in neutron stars with X-ray and ultraviolet observations. MNRAS 424, 93-103 (2012)

28. Gusakov M.E., Chugunov A.I., Kantor E.M.: Explaining observations of rapidly rotating neutron stars in LMXBs. arXiv:1305.3825 (2013)

29. Haskell B., Glampedakis K., Andersson N.: A new mechanism for saturating unstable r modes in neutron stars MNRAS 441, 1662-1668 (2014)

30. de Rham C., Gabadadze G. \& Tolley A.J.: Ghost free massive gravity in the Stckelberg language. Phys.Lett. 711, 190-195 (2012)

31. Hassan S.F. \& Rosen R.A.: Ghost-free massive gravity with a general reference metric. Journal of High Energy Physics 2, 26 (2012)

32. Gong Y.: Observational constraints on massive gravity. arXiv1210.5396 (2012)

33. Gratia P., Hu W. \& Wyman M.: Self-accelerating massive gravity: Exact solutions for any isotropic matter distribution. Phys.Rev. 86, 061504 (2012)

34. Abbott B.P., Abbott R., Acernese F., Adhikari R., Ajith P. et al.: Searches for Gravitational Waves from Known Pulsars with Science Run 5 LIGO Data. ApJ 713, 671-685 (2010)

35. Abadie J. et al.: Implementation and testing of the first prompt search for gravitational wave transients with electromagnetic counterparts. A\&A 539, A124-138 (2012)

36. Aasi J. et al.: First Searches for Optical Counterparts to Gravitational-wave Candidate Events. ApJ 211, 7-31 (2013)

37. Alford M.G. \& Schwenzer K.: Gravitational wave emission and spindown of young pulsars. arXiv:1210.6091 (2012) 


\section{University Library}

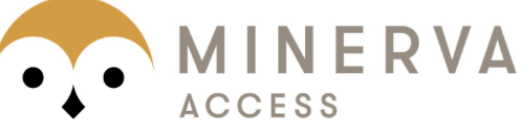

A gateway to Melbourne's research publications

Minerva Access is the Institutional Repository of The University of Melbourne

\section{Author/s:}

Branchesi, M;Woan, G;Astone, P;Bartos, I;Colla, A;Covino, S;Drago, M;Fan, X;Frasca, S;Hanna, C;Haskell, B;Hazboun, JS;Heng, IS;Holz, DE;Johnson-McDaniel, NK;Jones, ID;Keer, L;Klimenko, S;Kostas, G;Larson, SL;Mandel, I;Mapelli, M;Messenger, C;Mazzolo, G;Melatos, A;Mohanty, S;Necula, V;Normandin, M;Obara, L;Opiela, R;Owen, B;Palomba, C;Prodi, GA;Re, V;Salemi, F;Sidery, TL;Sokolowski, M;Schwenzer, K;Tiwari, V;Tringali, MC;Vedovato, G;Vousden, W;Yakushin, I;Zadrozny, A;Ziosi, BM

Title:

C7 multi-messenger astronomy of GW sources

Date:

2014-09-01

\section{Citation:}

Branchesi, M., Woan, G., Astone, P., Bartos, I., Colla, A., Covino, S., Drago, M., Fan, X., Frasca, S., Hanna, C., Haskell, B., Hazboun, J. S., Heng, I. S., Holz, D. E., JohnsonMcDaniel, N. K., Jones, I. D., Keer, L., Klimenko, S., Kostas, G. ,... Ziosi, B. M. (2014). C7 multi-messenger astronomy of GW sources. GENERAL RELATIVITY AND GRAVITATION, 46 (9), https://doi.org/10.1007/s10714-014-1771-6.

Persistent Link:

http://hdl.handle.net/11343/283230 